\title{
Transposition of European Guidelines for Energy Communities into Austrian Law: A Comparison and Discussion of Issues and Positive Aspects
}

\author{
Bernadette Fina ${ }^{1, *}$ and Hubert Fechner ${ }^{2}$ \\ 1 AIT Austrian Institute of Technology, Giefinggasse 4, 1210 Vienna, Austria \\ 2 Austrian Technology Platform Photovoltaic, Mariahilferstrasse 37-39, 1060 Vienna, Austria; h.fechner@tppv.at \\ * Correspondence: Bernadette.Fina@ait.ac.at; Tel.: +43-664-8839-0046
}

check for updates

Citation: Fina, B.; Fechner, H. Transposition of European Guidelines for Energy Communities into Austrian Law: A Comparison and Discussion of Issues and Positive Aspects. Energies 2021, 14, 3922. https://doi.org/10.3390/en14133922

Academic Editor: Attilio Converti

Received: 13 May 2021

Accepted: 25 June 2021

Published: 30 June 2021

Publisher's Note: MDPI stays neutral with regard to jurisdictional claims in published maps and institutional affiliations.

Copyright: (c) 2021 by the authors. Licensee MDPI, Basel, Switzerland. This article is an open access article distributed under the terms and conditions of the Creative Commons Attribution (CC BY) license (https:/ / creativecommons.org/licenses/by/ $4.0 /)$.

\begin{abstract}
The Renewable Energy Directive and the Electricity Market Directive, both parts of the Clean Energy for all Europeans Package (issued in 2019), provide supranational rules for renewable energy communities and citizen energy communities. Since national transpositions need to be completed within two years, Austria has already drafted corresponding legislation. This article aims at providing a detailed comparison of the European guidelines and the transposition into Austrian law. The comparison not only shows how, and to what extent, the European guidelines are transposed into Austrian law, but also helps to identify loopholes and barriers. The subsequent discussion of these issues as well as positive aspects of the Austrian transposition may be advantageous for legislators and policy makers worldwide in their process of designing a coherent regulatory framework. It is concluded that experts from different areas (i.e., project developers, scientists concerned with energy communities, energy suppliers and grid operators) should be closely involved in the law-making process in order to introduce different perspectives so that a consistent and supportive regulatory framework for energy communities is created.
\end{abstract}

Keywords: renewable energy communities and citizen energy communities; Clean Energy Package; transposition of European guidelines; comparison of European and national legislation; legislative barriers and issues; Austria

\section{Introduction}

In order to keep the increase in the global temperature below 2 degrees, the wellknown 20-20-20 goals were defined in 2007 and legally enacted in 2009 as part of the 2020 climate and energy package. Greenhouse gas emissions should be reduced by $20 \%$ from 1990 levels, 20\% of the European Union's energy should be generated from renewable sources and the energy efficiency should be improved by $20 \%$ [1]. Then, new goals were defined for 2030. Greenhouse gas emissions should be cut by $40 \%$ from the levels of 1990 , the share of renewable energy should be increased to $32 \%$ and energy efficiency by $32.5 \%$ [2]. Ultimately, by 2050, the EU aims to be climate neutral. This objective is the most important of the European Green Deal, and in line with the commitment to deliver on the Paris Agreement [3]. All these efforts to date have contributed to the next step in the transition process towards a sustainable energy future: the obligation for European member states to provide a regulatory framework to enable the establishment of renewable energy communities and citizen energy communities.

The Clean Energy for all Europeans Package (CEP)-a comprehensive update of the European energy policy framework enacted in 2019-represents a new attempt to meet the Paris Agreement commitment [4]. The eight legislative acts of the CEP comprise, amongst others, the Renewable Energy Directive (RED) [5] and the Electricity Market Directive (EMD) [6], providing new rules for energy communities in the European Union. Member states are given a time frame of a maximum two years to transpose the new rules into national law. 
As a result, the establishment of renewable energy communities (RECs) and citizen energy communities (CECs) will be legally enabled shortly.

Based on the European legislation, legislators published the first version of a comprehensive update of Austria's energy policy framework, covering multiple topics relating to renewable energy, in September 2020. This framework comprises, amongst others, rules for RECs and CECs, which are enacted in the novel Renewable Energy Expansion Act [7], as well as amendments of the existing Electricity Industry and Organisation Act [8]. Based on expert feedback, a revised version of the novel legislation was published in March 2021. However, adaptations concerning parts of the law that focus on energy communities are mainly of a structural nature (for example, parts of the provisions for RECs, originally contained in the Renewable Energy Expansion Act, were transferred to the Electricity Industry and Organisation $A c t)$, while the content remains mostly unchanged.

The first implementation in practice will reveal how feasible the new regulatory rules for energy communities in Austria actually are. However, in order to make predictions for the robustness of said rules, this article, in a first step, compares the European guidelines with the national transposition. In a second step, based on this comparison, potential barriers and other points of criticism are identified and discussed. In addition to the critical evaluation, positive aspects of the Austrian transposition are highlighted. The objective of this paper is twofold. Firstly, the Austrian attempt of transposing European directives into national law is made available to an international readership. Secondly, legislators in the European Union — and beyond - are encouraged to critically question and re-evaluate their countries' legislation on energy communities based on the issues identified in the Austrian legislation in order to avoid inconsistencies and barriers to implementation.

The remainder of this paper is structured as follows: Section 1.1 provides a comprehensive overview of the state of the art in the scientific literature and highlights the contribution of this article. The body of this article contains a comparison of the European guidelines and their transposition into Austrian law in Section 2. Section 3 discusses barriers and points of criticism as well as positive aspects in Sections 3.1 and 3.2, respectively. Conclusions and policy implications are provided in Section 4.

\subsection{State of the Art in Scientific Literature}

The state of the art in the scientific literature on energy communities in the context of the CEP is presented in Section 1.1.1. Subsequently, the development of energy communities in Austria from a legislative point of view is briefly presented in Section 1.1.2, before the contribution of this article is highlighted in Section 1.1.3.

\subsubsection{Energy Communities in the Context of the CEP}

The enactment of the CEP has prompted assessments of RECs and CECs from different angles. Different studies focus on RECs and CECs from a legal point of view. The legal status of energy communities under the CEP is addressed by [9]. Insights on the partial transposition of the RED in Italy are provided by [10], while [11] introduces the legal developments in the field of energy communities for the same country. Reference [12] elaborates on legal measures to aid profitability for energy communities and their participants. The authors of [13] analyze RECs under the CEP and highlight the need for an enabling framework to achieve widespread adoption. A cross-country comparison of the regulatory frameworks of nine countries is provided by [14] to reveal the main challenges and opportunities for different energy communities. The authors of [15] provide policy advice on regulating RECs and CECs in European countries, whereas [16] identifies barriers towards achieving a fair energy transition. Reference [17] tries to keep the expectations of the CEP towards community energy low and points out that multiple issues need to be addressed and clarified in the CEP's provisions for energy communities. Due to room for interpretation, there are concerns that the concept of RECs and CECs could be abused because of weak national transposition. 
The potential of energy communities to positively contribute to a sustainable energy future is assessed in different studies: Reference [18] assesses the greenhouse gas emission reduction potential, Reference [19] determines the cost-optimal economic potential, and the potential of RECs to contribute energy system transitions is elaborated on in [20]. However, the specific potential of RECs and CECs-energy community concepts under the CEP framework-have yet been assessed. Nevertheless, Reference [20] already found that the future advancement of energy communities depends on successfully linking them to established actors, as well as providing suitable legislation. Reference [21] identifies plausible scenarios for the possible development of energy communities under the CEP over the next decade. Furthermore, social acceptance of RECs is crucial for future deployment. The authors of [22] find that the social acceptance of local RECs significantly depends on the renewable energy technology. Trust is also an important point for the development, and the main motivations to participate seem to be concerns about environmental and climate issues [23]. Considering financial aspects, the authors of [24] compare the situation of eight different countries, concluding that RECs have the potential to reduce electricity costs. Evaluating the profitability of RECs under the framework of the RED transposed into Austrian law, Reference [25] finds that profitability strongly depends on additional community costs, such as a potential participation fee.

State support is crucial for the success of energy communities. The energy community concept should be accessible to all citizens from an administrative and organisational as well as from a financial point of view. This is confirmed by [17], who finds that for maximising the social potential of RECs and CECs, policy needs to ensure that all citizens can participate and benefit, especially those with limited financial means. To achieve optimal support, Reference [26] elaborates on the factors influencing REC formation and organisation. Going one step further, the authors of [27] present a methodology to support the decision-making process of citizens seeking to invest in RECs. Lastly, new business models that are applicable to a wide range of stakeholders need to be provided. For example, Reference [28] introduces consumer stock ownership plans as a prototype business model for RECs. This would also enable low-income households to invest in renewable projects since the RED obliges member states to facilitate participation for vulnerable customers, although the specific design of corresponding measures remains unclear [29].

\subsubsection{The Legislative Development of Energy Communities in Austria}

Before 2017, there was no legal possibility in Austria to establish energy communities, neither within the building border or within individual plots of land, nor between multiple buildings. (A certain grey area existed in the form of connecting buildings by establishing direct lines. However, the setting-up of sub-grid structures is not encouraged in Austria. Moreover, direct lines come with multiple restrictions, such as that common grounds are not allowed to be over-spanned.) The process of drafting a comprehensive regulatory framework for energy communities in Austria began in 2017 with the amendment of the Electricity Industry and Organisation Act [8]. This amendment allowed energy sharing within the borders of a single property, while the main intention was to enable the establishment of energy communities within individual multi-apartment buildings. However, energy communities on the single building level were only adopted occasionally, while other forms of communities (within a single property, e.g., between buildings of a business park) were not established at all [30]. This can most likely be traced back to citizens' unawareness, lack of state support, and legal uncertainties [30].

Due to the enforcement of the CEP and corresponding directives at European level in 2019, Austria, along with the other member states, is obliged to revise the current legislation so that establishing energy communities beyond the building border is possible. Regulatory rules concerning energy communities are laid down within the Electricity Industry and Organisation Act [8] and the Renewable Energies Expansion Act [7]. 


\subsubsection{Contribution of this Study}

The literature review shows that the regulatory framework for energy communities in different countries is of significant interest $([9-12,14,15,17])$ and that providing suitable legislation is crucial for the success of such concepts $([13,17,20])$. To this day, however, no detailed examination of a national transposition of the European directives has been made available to an international readership. Therefore, this article provides a detailed comparison of the European guidelines for RECs and CECs and the Austrian transposition into national law (status March 2021).

Based on the detailed comparison, legislative loopholes and barriers arising therefrom are identified, as well as positive aspects of the Austrian transposition. Highlighting both negative and positive aspects may be of significant importance for other legislators in the European Union and beyond in their process of developing a concise and unambiguous regulatory framework for energy communities.

\section{Comparison of the European Directives and the Austrian Transposition}

Sections 2.1 and 2.2 provide a detailed comparison of the European directives and the corresponding national transposition into Austrian law for RECs and CECs, respectively. It should be noted that this comparison is based on the Austrian legislation introduced in March 2021. Based on this comparison, barriers and issues as well as positive aspects, which will be discussed in Section 3, can be derived.

\subsection{Legislation for Renewable Energy Communities}

Table 1 presents a detailed comparison of the Austrian legislation on RECs and the European RED [5]. Rules for RECs are provided as part of the Renewable Energies Expansion Act (in German: Erneuerbaren Ausbau Gesetz-short form EAG) [7] and the amendment of the Electricity Industry and Organisation Act (in German: Elektrizitaetswirtschafts- und Organisationsgesetz-abbreviated to ElWOG) [8].

Table 1. Austrian legislation on Renewable Energy Communities (RECs) compared with the European guidelines of the Renewable Energy Directive [5] (RED).

\begin{tabular}{|c|c|}
\hline Austrian Legislation & Renewable Energy Directive \\
\hline $\begin{array}{l}\text { RECs are entitled to generate, consume, store, or } \\
\text { sell energy from renewable sources. In addition, } \\
\text { RECs are entitled to be active in aggregation and } \\
\text { provide other services. EAG } \$ 79(1)\end{array}$ & $\begin{array}{l}\text { '... renewable energy communities are entitled to produce, consume, store and sell renewable } \\
\text { energy, including through renewables power purchase agreements;' RED Article } 22 \text { ( } 2 \mathrm{a}) \\
\text { '... access all suitable energy markets both directly or through aggregation in a non- } \\
\text { discriminatory manner.' RED Article } 22(2 \mathrm{c}) \\
\text { '... renewable energy communities that supply energy or provide aggregation or other commer- } \\
\text { cial energy services ...' RED Article } 22(4 \mathrm{~b})\end{array}$ \\
\hline The free choice of supplier remains. EAG $\$ 79$ (1) & $\begin{array}{l}\text { 'Household consumers and communities engaging in renewables self-consumption should } \\
\text { maintain their rights as consumers, including the rights to have a contract with a supplier of } \\
\text { their choice and to switch supplier.' RED (72) }\end{array}$ \\
\hline $\begin{array}{l}\text { Members or partners of a REC are natural per- } \\
\text { sons, municipalities, public legal entities in re- } \\
\text { lation to local departments, other legal entities } \\
\text { under public law or SMEs. EAG } \$ 79(2)\end{array}$ & $\begin{array}{l}\text { '...the shareholders or members of which are natural persons, SMEs or local authorities, } \\
\text { including municipalities;' RED Article } 2(16 \mathrm{~b})\end{array}$ \\
\hline $\begin{array}{l}\text { A REC may be organised as an association, co- } \\
\text { operative, business partnership, corporation, or } \\
\text { similar association with legal personality. EAG } \\
\$ 79(2)\end{array}$ & $\begin{array}{l}\text { '..., it should be possible for Member States to choose any form of entity for renewable energy } \\
\text { communities,...' RED (71) }\end{array}$ \\
\hline $\begin{array}{l}\text { The primary purpose of a REC is not financial } \\
\text { gain, but to provide environmental, economic, or } \\
\text { social community benefits to its members or to } \\
\text { the areas in which it operates. EAG } \$ 79(2)\end{array}$ & $\begin{array}{l}\text { '... the primary purpose of which is to provide environmental, economic or social community } \\
\text { benefits for its shareholders or members or for the local areas where it operates, rather than } \\
\text { financial profits;' RED Article } 2 \text { (16c) }\end{array}$ \\
\hline
\end{tabular}


Table 1. Cont.

\begin{tabular}{l}
\hline Austrian Legislation \\
\hline Participation in a REC is voluntary and \\
open. In the case of private companies, \\
participation must not be their main \\
commercial or professional activity. EAG \\
$\S 79(2)$
\end{tabular}

The current status and development of RECs are to be evaluated, and unjustified obstacles or limitations to further development are to be identified. Furthermore, suggestions for improvement and the need for adaptation are to be evaluated. EAG §91 (3)

RECs may be subsidised. EAG $\S 80$

\section{Renewable Energy Directive}

'renewable energy community means a legal entity which, ..., is based on open and voluntary participation...' RED Article 2 (16a)

'To avoid abuse and to ensure broad participation, renewable energy communities should be capable of remaining autonomous from individual members and other traditional market actors that participate in the community as members or shareholders, or who cooperate through other means such as investment. Participation in renewable energy projects should be open to all potential local members based on objective, transparent and non-discriminatory criteria.' RED (71)

'Member States shall ensure that final customers, ..., are entitled to participate in a renewable energy community ... without being subject to unjustified or discriminatory conditions or procedures that would prevent their participation in a renewable energy community' RED Article 22 (1)

'Member States shall carry out an assessment of the existing barriers and potential of development of renewable energy communities in their territories.' RED Article $22(3)$

'Member States should ensure that renewable energy communities can participate in available support schemes on an equal footing with large participants. ... or allowing renewable energy communities to be remunerated through direct support where they comply with requirements of small installations.' RED (26)

\begin{abstract}
Generators that deliver electric energy to a grid in a local or regional area may participate in a REC, provided that they are not controlled by a utility, supplier, or power trader. ElWOG §16c (1)
\end{abstract}

The generation and consumption units within a REC must be connected via the low-voltage grid (local area) or the mediumvoltage grid (regional area) in the concession area of a grid operator. ElWOG §16c (2)

The transmission of energy via grid levels 1-4 or through the concession area of other grid operators is not permitted. ElWOG $\S 16 \mathrm{c}(2)$

Grid tariffs for RECs are determined based on which grid levels are used for electricity transfer. If only the low voltage grid is used, grid charges for grid level 7 arise; if the medium voltage grid is used as well, charges for grid levels 5, 6 and 7 are incurred. Costs of higher grid levels do not arise for electricity transfer within RECs. ElWOG $\S 52(2 a)$

By the end of the first quarter of 2024, the regulator must publish a cost-benefit analysis to determine whether appropriate and balanced participation of RECs in the system costs is ensured. EAG §79 (3) 'renewable energy community means a legal entity, which, ..., is effectively controlled by shareholders or members that are located in the proximity of the renewable energy projects...' RED Article 2 (16a)

'Member States may provide for renewable energy communities to be open to crossborder participation.' RED Article 22 (6)
'... community members should not be exempt from relevant costs, charges, levies and taxes that would be borne by final consumers who are not community members, producers in a similar situation, or where public grid infrastructure is used for those transfers.' RED (71)

'renewable energy communities are subject to ... cost-reflective network charges, as well as relevant charges, levies and taxes, ensuring that they contribute, in an adequate, fair and balanced way, to the overall cost sharing of the system in line with a transparent cost-benefit analysis of distributed energy sources developed by the national competent authorities;' RED Article 22 (4d) 
Table 1. Cont.

\begin{tabular}{l} 
Austrian Legislation \\
\hline Grid operators must be informed about the \\
establishment of a REC as well as about \\
the following elements and, if necessary, \\
changes to these elements: generation and \\
consumption facilities, metering point num- \\
bers, allocation of generated energy, alloca- \\
tion of non-consumed energy per 15 min, \\
admission and withdrawal of participating \\
network users, termination of the REC, and \\
dismantling of generation facilities. ElWOG \\
§16d (2)
\end{tabular}

\title{
Renewable Energy Directive
}

\begin{abstract}
A REC must conclude agreements that include at least the following elements: data management by the grid operator, operation, maintenance and servicing of generation facilities, liability, insurance. ElWOG $\S 16 \mathrm{~d}(3)$

RECs are entitled to own and to operate distribution networks. In this case, the rules applicable to distribution system operators apply. ElWOG §16d (4)
\end{abstract}

Grid users have a legal claim against network operators to participate in a REC. ElWOG §16d (1)

Grid users must be informed to which part

'... the relevant distribution system operator cooperates with renewable energy communities to facilitate energy transfers within renewable energy communities; of the distribution grid their consumption or generation facilities are connected within 14 days. ElWOG $\$ 16 \mathrm{c}(3)$

The grid operator must measure generation and consumption. If the consumption facilities are not equipped with smart meters, the grid operator must install them within two months. The measured values must be made available to the REC and the suppliers. ElWOG §16e (1)

The grid operator must allocate the agreed static or dynamic share of the generated energy to the grid users' respective plants. ElWOG §16e (3)

From 2022 onwards, consumption and generation units may participate in more than one energy community. ElWOG §111 (8)

‘Providing guidance to applicants ... by means of an administrative contact point is intended to reduce complexity for project developers and increase efficiency and transparency, including for renewables self-consumers and renewable energy communities. Such guidance is to be provided at an appropriate level... The single contact points should guide the applicant and facilitate through the entire administrative process ...' RED (50)

'Empowering jointly acting renewables self-consumers also provides opportunities for renewable energy communities to advance energy efficiency at household level and helps fight energy poverty through reduced consumption and lower supply tariffs.' RED (67)

'...the participation in the renewable energy communities is accessible to all consumers, including those in low-income or vulnerable households;' RED Article $22(4 \mathrm{f})$ 
Table 1. Cont.

\begin{tabular}{ll}
\hline Austrian Legislation & Renewable Energy Directive \\
\hline & 'Member States shall provide an enabling framework to promote and facilitate the \\
development of renewable energy communities. That framework shall ensure, inter \\
alia, that unjustified regulatory and administrative barriers to renewable energy \\
communities are removed;' RED Article 22 (4a)
\end{tabular}

\subsection{Legislation for Citizen Energy Communities}

Table 2 presents a detailed comparison of the Austrian legislation on CECs and the European EMD [6]. Rules for CECs are provided as part of the amendment of the Electricity Industry and Organisation Act (in German: Elektrizitaetswirtschafts- und Organisationsgesetzabbreviated to ElWOG) [8].

Table 2. Austrian legislation on Citizen Energy Communities (CECs) compared with the European guidelines of the Electricity Market Directive [6] (EMD).

Austrian Legislation
CECs are entitled to generate, consume, store, or sell
electrical energy. In addition, they are entitled to be
active in aggregation and to offer energy services to
their members. ElWOG $\$ 16 b(1)$

The free choice of supplier remains. ElWOG §16b (1)

\section{Electricity Market Directive}

'citizen energy community means a legal entity that may engage in generation, including from renewable sources, distribution, supply, consumption, aggregation, energy storage, energy efficiency services or charging services for electric vehicles or provide other energy services to its members or shareholders;' EMD Article 2 (11c)

'The provisions of this Directive on citizen energy communities provide for rights and obligations, which are possible to deduce from other, existing rights and obligations, such as the freedom of contract, the right to switch supplier,...' EMD (45)

\footnotetext{
Members or shareholders of a CEC are natural or legal persons and local authorities. ElWOG $§ 16 \mathrm{~b}(2)$
}

CECs may be organized as an association, cooperative, partnership, corporation, or similar association with legal personality. EIWOG §16b (2)

\footnotetext{
The main purpose of a CEC is not financial gain, but to provide environmental, economic or social community benefits to its members. ElWOG $\$ 16 b(2)$
}

Participation in CECs is voluntary and open

ElWOG $\$ 16 b(2)$

\footnotetext{
Control within a CEC is limited to natural persons, local authorities, and small companies, provided that they do not perform the function of an electricity supplier. Control is established if the majority is held by the aforementioned members. ElWOG $§ 16 \mathrm{~b}$ (3)

Grid users have a legal claim against network operators to participate in a CEC. ElWOG §16d (1)

Grid operators must be informed about the establishment of a CEC as well as about the following elements and, if necessary, changes to these elements: generation and consumption facilities, metering point numbers, allocation of generated energy, allocation of nonconsumed energy per $15 \mathrm{~min}$, admission and withdrawal of participating network users, termination of the CEC, and dismantling of generation facilities. ElWOG $\S 16 \mathrm{~d}(2)$
}

'citizen energy community means a legal entity that is ... effectively controlled by members or shareholders that are natural persons, local authorities, including municipalities, or small enterprises.' EMD Article 2 (11a)

'Membership of citizen energy communities should be open to all categories of entities.... It should therefore be possible for Member States to provide that citizen energy communities take any form of entity, for example that of an association, a cooperative, a partnership, a non- profit organisation or a small or medium-sized enterprise' EMD (44)

'citizen energy community means a legal entity that has for its primary purpose to provide environmental, economic or social community benefits to its members or shareholders or to the local areas where it operates rather than to generate financial profits;' EMD Article $2(11 b)$

'Household customers should be allowed to participate voluntarily in community energy initiatives as well as to leave them...' EMD (43)

'citizen energy community means a legal entity that is based on voluntary and open participation...' EMD Article 2 (11a)

... participation in a citizen energy community is open and voluntary; members or shareholders of a citizen energy community are entitled to leave the community,...' EMD Article $16(1 \mathrm{a}, 1 \mathrm{~b})$

'..., the decision- making powers within a citizen energy community should be limited to those members or shareholders that are not engaged in large-scale commercial activity and for which the energy sector does not constitute a primary area of economic activity.' EMD (44)

'Access to a citizen energy community's network should be granted on fair and costreflective terms.' EMD (43) 
Table 2. Cont.

\begin{tabular}{l} 
Austrian Legislation \\
\hline A CEC must conclude agreements that include at \\
least the following elements: data management \\
by the grid operator, operation, maintenance and \\
servicing of generation facilities, liability, insur- \\
ance. ElWOG §16d (3)
\end{tabular}

CECs are entitled to own and to operate distribution networks. In this case, the rules applicable to distribution system operators apply. ElWOG §16d (4)

\section{Electricity Market Directive}

The grid operator must measure generation and consumption. If the consumption facilities are not equipped with a smart meter, the grid operator must install them within two months. The metered values must be made available to the suppliers and the CEC. ElWOG §16e (1)

In addition, the metered values of a grid operator must be made available to all other grid operators in whose concession areas the CEC's generation and consumption facilities are located. ElWOG §16e (2)

The grid operator must allocate the agreed static or dynamic share of the generated energy to the grid users' respective plants. ElWOG §16e (3)

The current status and development of CECs must be evaluated, and unjustified obstacles or limitations to further development are to be identified. Furthermore, suggestions for improvement and the need for adaptation are to be evaluated. EAG, $\S 91$ (3)
'This Directive empowers Member States to allow citizen energy communities to become distribution system operators either under the general regime or as 'closed distribution system operators'. Once a citizen energy community is granted the status of a distribution system operator, it should be treated as, and be subject to the same obligations as, a distribution system operator.' EMD (47) 'Member States may decide to grant citizen energy communities the right to manage distribution networks in their area of operation...' EMD Article 16 (4)

'... relevant distribution system operators cooperate with citizen energy communities to facilitate electricity transfers within citizen energy communities;' EMD Article 16 (1d)

From 2022 onwards, consumption and generation units may participate in more than one energy community. ElWOG $§ 111(8)$

'The regulatory authority shall have the following duties: monitoring the removal of unjustified obstacles to and restrictions on the development of consumption of self- generated electricity and citizen energy communities' EMD Article 59 (1z)

'Community energy can also advance energy efficiency at household level and help fight energy poverty through reduced consumption and lower supply tariffs.' EMD (43)

'Member States may provide in the enabling regulatory framework that citizen energy communities are open to cross-border participation' EMD Article 16 (2a)

\section{Points of Criticism and Positive Aspects of the Austrian Transposition}

This section identifies points of criticism (Section 3.1) as well as positive aspects (Section 3.2) of the Austrian transposition of the European Directives into national law.

\subsection{Barriers and Points of Criticism of the Austrian Transposition}

Based on the comparison of European guidelines and the transposition into Austrian law, barriers and points of criticism are outlined and discussed in the following.

\subsubsection{Reduction of Grid Tariffs as Incentive/Support for RECs}

RECs in Austria will be incentivised, amongst other things, by a reduction of grid tariffs for electricity transfer between participants. If only the low voltage grid is used for electricity transfer, grid tariffs are reduced significantly. If the medium voltage grid is used 
as well, grid tariffs are still reduced but to a lesser extent. (The exact numbers have not yet been published; however, a reduction of $50-60 \%$ is expected for RECs that operate within the low-voltage grid and $30-40 \%$ for RECs also using the medium-voltage grid.) This political decision is questionable, since it is undetermined whether RECs actually reduce the grid burden, which would justify such decision. If RECs pose a burden to the grid, concerns over unfair distribution of grid costs arise. Therefore, the regulator (E-Control) is required by law to publish, by the end of the first quarter of 2024, a cost-benefit analysis to determine whether an appropriate and balanced participation of RECs in the system costs is ensured (EAG \$79 (3)). According to the annotations of the EAG [31], p. 19, it is to be decided whether adaptations of existing provisions are necessary based on the cost-benefit analysis.

The possibility of a change in grid tariffs for electricity transfer within RECs in 2024 causes uncertainty amongst project developers and potential participants. Although it is not the primary purpose of RECs to generate financial benefits, increasing the profitability of REC participation by reducing grid tariffs or other financial benefits is important to citizens with limited financial means.

The reduction of grid tariffs is a topic of controversy not only from the perspective of an adequate and balanced participation of RECs in the system costs, but also from the viewpoint of participants in different federal provinces. Grid charges will be reduced by a fixed percentage throughout Austria, despite the fact that grid charges differ in the federal provinces. (This is due to the different grid situations in the individual federal provinces. For example, grid development differs significantly for densely built city areas compared to rural areas. Moreover, mountain regions have different grid specifications compared to a flat landscape. The grid tariffs reflect the actual costs for the grid operators; Those costs are scrutinised by the regulator, E-control, and based thereon, according grid tariffs are set.) as a result, participants in federal provinces with lower grid tariffs feel disadvantaged.

In conclusion, despite the intention to incentivise local electricity transfer, it might have been more convenient to introduce grid-independent means of support, such as tax exemptions, or premiums for energy transfer within a REC.

\subsubsection{Changes in the Grid Structure}

Since participants in RECs need to be connected via the low voltage grid or the medium voltage grid, problems may arise when grid operators change the grid structure after a REC has been established. In the annotations to the legislation ([31], pp. 27, 28) it is explicitly stated that retrospective technical changes of the distribution grid shall not affect established RECs. However, annotations are not legally binding. This is cause for concern amongst potential REC developers and participants since it is undetermined whether-and to what extent- this legislative loophole might affect existing communities.

In conclusion, the current version of the Austrian legislation needs to be updated such that the according passage is transferred from the annotations to the law itself to ensure that retrospective changes in the grid structure prevent RECs from being terminated.

\subsubsection{Open Participation in RECs and CECs}

The RED [5] and the EMD [6] as well as the national transpositions emphasise that the participation in RECs and CECs is voluntary and open. While there is no doubt about what is meant by voluntary participation, the term "open" leaves room for speculation and raises unanswered questions from the viewpoint of (i) potential new participants, (ii) the energy community itself as well as established participants, and (iii) community founders.

(i) Can a citizen who applies for participation in a certain energy community be rejected? Given the requirement of a non-discriminatory and open participation, the impression may arise that a community may not be entitled to reject a potential participant.

(ii) Can a REC or CEC reject participants based on solid grounds? Rejecting a potential new participant does not necessarily constitute an act of discrimination. If an energy community is, for example, precisely planned such that the generated energy is 
optimally used within the community, it is possible that including another member may have a negative impact on the existing participants.

(iii) Are not the statutes of the chosen legal personality decisive as to who may participate? If not, this might lead to the problem that energy communities are scarcely adopted due to the impression that establishing an energy community imposes multiple obligations but only confers limited rights.

In the RED (71) it is stated that 'Participation ... should be open to all potential local members based on objective, transparent and non-discriminatory criteria'. From this statement, it can be derived that European legislators suggest that a potential participant can only be rejected on solid grounds, as suggested in (ii) above. This interpretation is in line with [17], stating that as long as new members are accepted, RECs should not be able to exclude anyone based on arbitrary criteria. However, it is also stated that the appropriate level of openness should depend upon the context. In that case, on the one hand, clear discrimination of potential participants would be avoided, and, on the other hand, the founder or responsible party for an energy community does not entirely lose their decision-making power. However, there is the need for clarification of the term "open" in the Austrian transposition.

\subsubsection{Generation Units-Operated and Controlled by the Community}

Generation units, whether jointly or privately owned, are to be operated and controlled by the community (except for the share used for self-consumption). However, similar to the issue raised in Section 3.1.2, requirements for generation units to be controlled and operated by the energy community are only contained in the annotations ([31] p. 19 for RECs and p. 26 for CECs) and are therefore not legally binding.

However, a provision that is contained in the legislation itself (EIWOG §111 (8)) requires that from 2022 onwards, consumption as well as generation units are entitled to participate in more than one energy community. This possibility, in combination with the requirement for generation units to be effectively controlled by the energy community, may cause significant difficulties in its implementation. Questions such as which energy community would take priority remain unanswered.

To conclude, it can be remarked that provisions concerning the operation and control of generation units need to be contained in the actual legislation rather than the annotations. Otherwise, some participants may refuse to assign the rights of controlling their private generation unit to the community due to missing legislative grounds, which would significantly obstruct a fast and simple energy community roll-out.

\subsubsection{Participation in More Than One Energy Community}

According to ElWOG $\S 111$ (8), it will be possible for consumption and generation units to participate in more than one energy community from 2022 onward. As already mentioned in Section 3.1.4, this provision leaves multiple questions unanswered, which might cause potential administrative barriers. It must be determined which energy community primarily supplies a customer who is a participant in several energy communities, and in which order the other energy communities deliver energy to this customer. The same accounts for generation units. It must be determined in which order a generation unit supplies different energy communities in case of participating in multiple communities. This situation might require not only contracts within an energy community, but also contracts between different energy communities in order to set a certain prioritisation in stone. In addition, the grid operator would need to be provided with such information in order to properly allocate electricity as requested by the communities based on individual distribution keys.

In conclusion, it is recommended to withdraw the option of participating in multiple energy communities, at least for the time being, in order to avoid adding administrative complications to an already complex situation. 


\subsubsection{REC Legislation Tailored to the Electricity Sector}

Since the concept of RECs, in contrast to CECs, is not restricted to the electricity sector, the national transposition of the RED needs to be neutral towards the energy carrier. However, the annotations of [31], p.18, state that the concept of self-supply and community supply primarily concerns the area of renewable electricity, which is why the national transposition is currently tailored to the electricity sector.

Although it is true that REC initiatives are mainly undertaken in the electricity sector, there are also ambitions to establish RECs in the heating sector. The fact that the legislation is tailored to the electricity sector may become a problem in the medium-term, when the implementation of projects with renewable heat sharing is undertaken. Therefore, it is recommended that the applicability of the drafted legislation to RECs including the heating sector is investigated. If proven necessary, amendments to the legislation need to be swiftly enacted.

\subsubsection{Energy Poverty in the Context of RECs and CECs}

Energy poverty is a topic that is highlighted more than once in the European Directives (e.g., RED (67), RED Article 22 (4f), EMD (43)). This important aspect is completely neglected in the Austrian legislation. Without explicit efforts towards including less privileged citizens, energy communities may remain concepts for affluent citizens.

In order to make participation in energy communities available to all citizens, a certain economic viability is indispensable. For this reason, the uncertainty resulting from a possible change in the reduced grid tariffs (Section 3.1.1) from 2024 onwards is even more serious, which supports the conclusion that on the one hand, additional support schemes for energy communities in general (currently, no support is foreseen for CECs) should be introduced, while on the other hand, support schemes that are tailored to vulnerable customers need to be proposed as well.

\subsection{Positive Aspects of the Austrian Transposition}

However, there are also positive aspects of the Austrian transposition that are worth highlighting.

\subsubsection{Interaction of Grid Operators and Energy Communities}

A lot of effort was put into defining clear rules for the interaction between grid operators and energy communities. This is crucial because grid operators may be reluctant when it comes to cooperating with energy communities due to additional efforts such as measurement and electricity allocation. However, if grid operators commit to cooperating with energy communities, clear rules that define the obligations of energy communities towards the grid operators also need to be established.

\subsubsection{Operation of Distribution Grids for RECs and CECs}

Unlike the RED, the Austrian legislation stipulates that RECs can own as well as operate distribution networks. Thus, in Austria, RECs as well as CECs are authorised to assume the rights and obligations of a grid operator. Although energy communities operating distribution grids may remain an exception, it is an important step towards potential competition that may incentivise grid operators to increase cooperation with energy communities.

\subsubsection{Grid Levels as Proximity Criterion for RECs}

In Austria, grid levels are used to define the proximity of REC participants. Since not only the low-voltage grid but also the medium-voltage grid can be used for electricity transfer, RECs are restricted as little as possible. Moreover, using grid levels to geographically constrain RECs appears to be a decent solution compared to other options such as defining a certain perimeter or using postal codes, which might disadvantage citizens in remote areas or small municipalities. 


\subsubsection{Official Body to Administratively Support RECs and CECs}

The RED explicitly states that guidance is to be provided by means of an administrative contact point with the intention of reducing complexity for project developers of RECs (RED (50)). Such guidance is not explicitly enacted in the Austrian legislation; however, an official body that provides administrative support and guides the establishment of energy communities is currently being established on a national level as well as on a federal province level. This official body is called Österreichische Koordinationsstelle für Energiegemeinschaften, which can be translated as Austrian Body for the Coordination of Energy Communities. This official body will offer services for citizens and project developers with different levels of knowledge in order to make the concept of energy communities accessible for everybody. Services comprise answering administrative questions, providing a knowledge base as well as guidance through the individual steps of founding, and setting up an energy community.

\subsubsection{Additional Support for RECs and CECs}

Besides the reduced grid charges, RECs are incentivised by being exempt from levies such as the renewable energy surcharge ([7] EAG \$75 (5)) and the electricity surcharge [32]. (CECs, on contrary, will not receive similar financial support. It might be the case that individual federal provinces implement their own support schemes for energy communities in the future. However, there is no information about further state support for energy communities yet.)

\section{Discussion and Conclusions}

This article aims to provide a detailed comparison of the European guidelines-the Renewable Energy Directive and the Electricity Market Directive-and the Austrian transposition concerning Renewable Energy Communities and Citizen Energy Communities. The comparison not only shows how and to what extent the guidelines are transposed but also provides an opportunity to identify loopholes and barriers. The discussion of such issues as well as positive aspects of the Austrian transposition may be helpful for other countries' legislators and policy makers.

The comparison of European Directives and national law broadly reveals two things: On the one hand, Austrian legislators managed to integrate most European requirements for RECs and CECs into national law. On the other hand, there are some issues in the transposition that have not yet been comprehensively examined. An example is the reduced grid tariffs for RECs: if RECs reduce the grid burden, reducing grid tariffs is a valid additional incentive for local energy consumption. However, at this point, tariff reductions are granted without any scientific basis. Therefore, after evaluation in 2024, grid tariff reductions may be scaled down or even canceled, which causes uncertainty amongst project developers and may result in discouraging the adoption of RECs. Another example is the provison allowing generation and consumption units to take part in multiple energy communities. The interaction of energy communities with traditional market players and their billing and accounting processes are already complex. Increasing the complexity by adding such a provision without explicit guidance on how this could be actually implemented is not recommended, at least in the first phase of energy community roll-out.

From the issues identified in the Austrian legislation, it can be deduced that complex topics such as energy communities cannot be fully grasped in all their facets by policy makers themselves. Therefore, experts of all relevant fields (i.e., project developers, scientists, energy suppliers, and grid operators) need to be closely involved in the law-making process in order to prevent barriers from arising. Since Austria is one of the first countries to draft legislation based on the European directives, legislators of other countries may learn from the discussion provided in this study and avoid similar inconsistencies in their own legislation.

For future work, it may be of international interest if other countries also elaborated on their national energy community legislation and discussed issues and advantages. This 
would facilitate knowledge transfer between countries and help to continuously improve laws in order to successfully establish the concept of energy communities throughout the European Union and beyond.

Author Contributions: The authors contributed to this work as follows: B.F.: Conceptualization, methodology, validation, formal analysis, investigation, data curation, writing —original draft preparation, writing — review and editing. H.F.: validation, writing — review All authors have read and agreed to the published version of the manuscript.

Funding: This work was developed in the project CLUE (FFG no. 872286). As such, this project has received funding from the European Union's Horizon 2020 research and innovation programme under grant agreement no. 775970 .

Conflicts of Interest: The authors declare no conflict of interest. The funders had no role in the design of the study; in the collection, analyses, or interpretation of data; in the writing of the manuscript; or in the decision to publish the results.

\author{
Abbreviations \\ The following abbreviations are used in this manuscript: \\ CEC Citizen Energy Community \\ CEP Clean Energy for All Europeans Package \\ EAG Erneuerbaren Ausbau Gesetz (English: Renewable Energies Expansion Act) \\ ElWOG Elektrizitaetswirtschafts- und Organisationsgesetz \\ (English: Electricity Industry and Organisation Act) \\ EMD Electricity Market Directive \\ REC Renewable Energy Community \\ RED Renewable Energy Directive
}

\title{
References
}

1. European Commission. 2020 Climate and Energy Package; European Commission: Brussels, Belgium, 2021.

2. European Commission. 2030 Climate and Energy Framework; European Commission: Brussels, Belgium, 2021.

3. European Commission. 2050 Long-Term Strategy; European Commission: Brussels, Belgium, 2021.

4. European Commission. Clean Energy for All Europeans Package; European Commission: Brussels, Belgium, 2019.

5. EUR-Lex. Directive (EU) 2018/2001 of the European Parliament and of the Council of 11 December 2018 on the Promotion of the Use of Energy from Renewable Sources. 2018. Available online: https:/ / eur-lex.europa.eu/legal-content/EN/TXT/?uri=uriserv: OJ.L_.2018.328.01.0082.01.ENG\&toc=OJ:L:2018:328:TOC (accessed on 29 June 2021).

6. EUR-Lex. Directive (EU) 2019/944 of the European Parliament and of the Council of 5 June 2019 on Common Rules for the Internal Market for Electricity and Amending Directive 2012/27/EU. 2019. Available online: https: / / eur-lex.europa.eu/legalcontent/en/TXT/?uri=CELEX:32019L0944 (accessed on 29 June 2021).

7. BMK. EAG-Entwurf 2021-03-16. In BMK Bundesministerium fuer Klimaschutz, Umwelt, Energie, Mobilitaet, Innovation und Technologie; BMK: Vienna, Austria, 2021.

8. RIS. Elektrizitaetswirtschafts- und orGanisationsgesetz 2010. Rechtsinformationssystem des Bundes. 2010. Available online: https: / /www.ris.bka.gv.at/GeltendeFassung.wxe?Abfrage=Bundesnormen\&Gesetzesnummer=20007045 (accessed on 29 June 2021 ).

9. Gerasina, O. Energy Communities in the Centre of EU Energy Transition. Legal Statusof Energy Community under the Clean Energy for all Europeans Package. 2020. Available online: https:/ / erepo.uef.fi/bitstream/handle/123456789/22938/urn_nbn_fi_ uef-20200856.pdf?sequence $=-1$ (accessed on 29 June 2021).

10. Zulianello, M.; Angelucci, V.; Moneta, D. Energy Community and Collective Self Consumption in Italy. In Proceedings of the 2020 55th International Universities Power Engineering Conference (UPEC), Turin, Italy, 1-4 September 2020. [CrossRef]

11. Candelise, C.; Ruggieri, G. Status and Evolution of the Community Energy Sector in Italy. Energies 2020, 13, 1888. [CrossRef]

12. Cejka, S. Legal measures to aid profitability for energy communities and their participants. In Proceedings of the 2020 Zooming Innovation in Consumer Technologies Conference (ZINC), Novi Sad, Serbia, 26-27 May 2020. [CrossRef]

13. Lowitzsch, J.; Hoicka, C.; van Tulder, F. Renewable energy communities under the 2019 European Clean Energy Package Governance model for the energy clusters of the future? Renew. Sustain. Energy Rev. 2020, 122, 109489. [CrossRef]

14. Inês, C.; Guilherme, P.L.; Esther, M.G.; Swantje, G.; Stephen, H.; Lars, H. Regulatory challenges and opportunities for collective renewable energy prosumers in the EU. Energy Policy 2020, 138, 111212. [CrossRef]

15. Sokołowski, M.M. Renewable and citizen energy communities in the European Union: How (not) to regulate community energy in national laws and policies. J. Energy Nat. Resour. Law 2020, 38, 289-304. [CrossRef] 
16. Heldeweg, M.A.; Saintier, S. Renewable energy communities as 'socio-legal institutions': A normative frame for energy decentralization? Renew. Sustain. Energy Rev. 2020, 119, 109518. [CrossRef]

17. Roberts, J. Power to the people? Implications of the Clean Energy Package for the role of community ownership in Europe's energy transition. Rev. Eur. Comp. Int. Environ. Law 2020, 29, 232-244. [CrossRef]

18. Schram, W.; Louwen, A.; Lampropoulos, I.; van Sark, W. Comparison of the Greenhouse Gas Emission Reduction Potential of Energy Communities. Energies 2019, 12, 4440. [CrossRef]

19. Fina, B.; Auer, H.; Friedl, W. Cost-optimal economic potential of shared rooftop PV in energy communities: Evidence from Austria. Renew. Energy 2020, 152, 217-228. [CrossRef]

20. Dóci, G.; Vasileiadou, E.; Petersen, A.C. Exploring the transition potential of renewable energy communities. Futures 2015, 66, 85-95. [CrossRef]

21. Verde, S.; Rossetto, N. The future of renewable energy communities in the EU: An investigation at the time of the Clean Energy Package. 2020. Available online: https:/ / cadmus.eui.eu/handle/1814/68383 (accessed on 29 June 2021).

22. Azarova, V.; Cohen, J.; Friedl, C.; Reichl, J. Designing local renewable energy communities to increase social acceptance: Evidence from a choice experiment in Austria, Germany, Italy, and Switzerland. Energy Policy 2019, 132, 1176-1183. [CrossRef]

23. Soeiro, S.; Dias, M.F. Renewable energy community and the European energy market: Main motivations. Heliyon 2020,6 , e04511. [CrossRef] [PubMed]

24. Radl, J.; Fleischhacker, A.; Revheim, F.H.; Lettner, G.; Auer, H. Comparison of Profitability of PV Electricity Sharing in Renewable Energy Communities in Selected European Countries. Energies 2020, 13, 5007. [CrossRef]

25. Fina, B.; Auer, H. Economic Viability of Renewable Energy Communities under the Framework of the Renewable Energy Directive Transposed to Austrian Law. Energies 2020, 13, 5743. [CrossRef]

26. Dóci, G. Collective Action with Altruists: How Are Citizens Led Renewable Energy Communities Developed? Sustainability 2021, 13, 507. [CrossRef]

27. Moncecchi, M.; Meneghello, S.; Merlo, M. Energy Sharing in Renewable Energy Communities: The Italian Case. In Proceedings of the 2020 55th International Universities Power Engineering Conference (UPEC), Turin, Italy, 1-4 September 2020. [CrossRef]

28. Lowitzsch, J. Consumer Stock Ownership Plans (CSOPs)—The Prototype Business Model for Renewable Energy Communities. Energies 2019, 13, 118. [CrossRef]

29. Hanke, F.; Lowitzsch, J. Empowering Vulnerable Consumers to Join Renewable Energy Communities-Towards an Inclusive Design of the Clean Energy Package. Energies 2020, 13, 1615. [CrossRef]

30. Klima- und Energiefonds. Evaluierung der bisherigen Erfahrungen bei der Umsetzung von PV-Gemeinschaftsanlagen nach §16a ElWOG. 2021, not yet published.

31. BMK. Erlaeuterungen zum EAG 2021-03-16. In BMK Bundesministerium fuer Klimaschutz, Umwelt, Energie, Mobilitaet, Innovation und Technologie; BMK: Vienna, Austria, 2021.

32. BMK. ElAbgG-UmsetzungsV; BMK: Vienna, Austria, 2021; Available online: https://www.ris.bka.gv.at/Dokumente/BgblAuth/ BGBLA_2021_II_82/BGBLA_2021_II_82.html (accessed on 29 June 2021). 